\section{La extensión universitaria y el nuevo plan de estudios de la carrera de Abogacía en la Universidad Nacional de La Plata}

\section{Cristian Furfaro}

Docente investigador de la Universidad

Nacional de La Plata - Consejo

Nacional de Investigaciones Científicas

y Tecnológicas (CONICET), Argentina.

cfuraro@hotmail.com
Integración de la docencia y la extensión /

Desafíos de gestión

RECEPCIÓN: 24/06/16

ACEPTACIÓN FINAL: 10/10/16

\section{Resumen}

El objetivo del presente artículo es reflexionar en torno a la inclusión de la extensión universitaria en el nuevo plan de estudios de la carrera de Abogacía de la Facultad de Ciencias Jurídicas y Sociales (FCJyS) de la Universidad Nacional de La Plata (UNLP). Iniciaremos este recorrido con una breve descripción de algunas características de la extensión universitaria en la FCJyS, su relación con el plan de estudios vigente y el proceso de sanción del nuevo plan de estudios. Posteriormente, estableceremos algunas cuestiones teóricas que guiarán el análisis de los documentos elaborados por la FCJyS y que nos permitirán reflexionar sobre los fundamentos esgrimidos para incluir la extensión universitaria en el nuevo plan de estudios, los principios sostenidos y las decisiones que se tomaron. Finalmente, explicitaremos algunas cuestiones respecto de la tensión que existe en el mundo del Derecho entre prácticas profesionales y extensión universitaria.

Palabras-clave

- Extensión universitaria

- Derecho

- Plan de estudios

- Prácticas profesionales

\section{Resumo}

O objetivo do presente artigo é refletir a respeito da inclusão da extensão universitária na nova estrutura curricular do curso de Direito da Facultad de Ciencias Jurídicas y Sociales (FCJyS) da Universidad Nacional de La Plata (UNLP). Começaremos este percurso com uma breve descrição de algumas características da extensão universitária na FCJyS, a relação com a estrutura curricular atual, e o processo de aprovação da nova estrutura curricular. A seguir, estabeleceremos algumas questões teóricas que vão orientar a análise dos documentos elaborados pela FCJyS, e que nos vão permitir refletir sobre os fundamentos esgrimidos para incluir a extensão universitária na nova estrutura curricular, os princípios defendidos e as decisões que foram tomadas.

Finalmente, explicitaremos algumas questões relativas à tensão que existe no mundo do Direito entre práticas profissionais e extensão universitária.

Palavras-chave

- Extensão universitária

- Direito

- Estrutura curricular

- Práticas profissionais
-

Furfaro, C. (2016). La extensión universitaria y el nuevo plan de estudios de la carrera de abogacía en la Universidad Nacional de La Plata. En Revista $+E$ versión digital, (6), pp. 172-179. Santa Fe, Argentina: Ediciones UNL 
1. Sobre los límites del presente trabajo y el camino a recorrer El presente artículo es apenas un primer paso, una aproximación, al análisis del nuevo plan de estudios de la carrera de abogacía de la Facultad de Ciencias Jurídicas y Sociales (en adelante, la Facultad) de la UNLP. La breve reflexión aquí presentada se elaboró con base en el análisis de los documentos sobre el nuevo plan de estudios que la Facultad publicó en la primera semana del mes de mayo de 2016. ${ }^{1}$ Esos documentos solo contienen la estructura del plan de estudios y su fundamentación. La lectura de las Actas del Honorable Consejo Directivo (HCD) nos ha permitido identificar a los actores claves del proceso, pero no han aportado mayores datos en lo que respecta al análisis de los debates suscitados ni sobre los posicionamientos específicos de los diferentes claustros. En razón de ello, el lector encontrará, como resultado de este primer y muy reciente análisis, una única voz, la voz institucional de la Facultad, y sus cambios, que se reflejan en las modificaciones que la propuesta de reforma recibió para convertirse en el nuevo plan de estudios.

La deconstrucción del nuevo plan, nos encuentra trabajando en la realización de entrevistas a informantes clave y actores institucionales. Estas entrevistas nos permitirán explicitar - en futuros trabajos - qué debates se suscitaron al interior de la Facultad, qué papel jugó cada uno de los actores en la dinámica institucional y qué aspectos e intereses primaron al momento dar forma al nuevo plan de estudios. Y así, especificaremos, presentando las múltiples voces heteroglóticas que habitan la Facultad, cuáles fueron los debates, conflictos y tensiones que conviven - y convivieron - en la voz institucional, esa voz que como un todo, hemos presentado en el presente artículo.

\section{Extensión universitaria y planes de estudio en la FCJyS}

La extensión universitaria en la Facultad se compone de actividades de diferente tipo: culturales, sociales, de asistencia técnica, de desarrollo tecnológico, de difusión, de capacitación, y de formación profesional, entre otras. ${ }^{2}$ Desde hace un tiempo, la actividad extensionista de la Facultad - y de la mayoría de las facultades de Derecho- se ha canalizado a través de los Consultorios Jurídicos Gratuitos. Este espacio funciona en la FCJyS desde 1993 y tiene dos objetivos centrales: 1) facilitar el acceso a la justicia de sectores sociales desprotegidos; y 2) abarcar las dificultades que aquejan a los estudiantes avanzados y graduados recién recibidos en cuanto a la ausencia de práctica efectiva en el ejercicio profesional. La idea de concebir los Consultorios Jurídicos Gratuitos como espacios de extensión universitaria y práctica profesional no ha tenido influencia sobre el plan de estudios vigente. Ni su matriz básica que data de 1953, ni las reformas introducidas en 1980 y 1989, contemplaron la realización de actividades de extensión universitaria entre las exigencias curriculares, como así tampoco la inclusión de los consultorios en el esquema actual de prácticas profesionales de la carrera. ${ }^{3}$

Entre los años 2007 y 2010, la Facultad llevó adelante un proceso de Autoevaluación Académico-Institucional, a partir del cual se dispone, mediante la Resolución n 198/11 del HCD, iniciar un programa para el análisis, debate y formulación de propuestas tendientes a la reforma del plan de estudios de la carrera de abogacía. ${ }^{4}$ El día 27 de diciembre de 2013, el HCD, por Resolución n³36/13, resuelve aprobar una propuesta de reforma del plan de estudios. El documento que acompañaba la resolución
1) Los documentos mencionados pueden obtenerse en la página de la Facultad http://www.jursoc.unlp.edu. ar/nuevo-plan-de-estudio-mainuevo. 2) Las actividades de la Secretaría de Extensión Universitaria de la FCJyS pueden observarse en http://www. jursoc.unlp.edu.ar/index.php/noticiasextension

3) El Plan de Estudios vigente contiene 31 asignaturas teóricas, 2 asignaturas prácticas y 1 seminario electivo. Las asignaturas prácticas son la Adaptación Profesional de Procedimientos Civiles y Penales, y la Adaptación Profesiona de Procedimientos Civiles y Comerciales. En ambos casos es el docente quien decide si el estudiante desarrolla prácticas en terreno o sólo elabora los escritos requeridos.

4) El debate y elaboración de propuestas se estructuró en seis comisiones que se reunieron durante un año, recibieron propuestas y elaboraron proyectos. Las comisiones fueron: 1) Materias iniciales o propedéuticas, 2) Derecho Público, 3) Derecho Privado, 4) Perfil profesional, perfil curricular y enseñanza del Derecho, 5) Ciencias Sociales y áreas de conocimiento complementarias para la formación del abogado y 6 ) Prácticas Profesionales. Cada una fue coordinada por un consejero directivo e integrada por los profesores de cada área y todo integrante de los claustros que quisiera sumarse. Se recibía todo tipo de propuestas: desde e-mails con comentarios y puntos de vista sobre determinados aspectos a proyectos concretos de creación de materias, con indicación de correlatividades, contenidos mínimos, programa de enseñanza, etc. De la recopilación y sistematización de las propuestas se elaboró un Informe Final que una Comisión Redactora presentó al Consejo Directivo y que constituyó la base de la propuesta institucional. Como integrante del proyecto de investigación UNLP J101 "Las nuevas configuraciones del campo de la formación del abogado en Argenti- na: instituciones, planes de estudio y prácticas profesionales", participé de la elaboración de un informe. En él se destacaba la necesidad de incluir en el Plan de Estudios la realización de actividades de extensión universitaria que, apoyadas en fundamentos teórico-metodológicos, permita a cada uno de los participantes reflexionar sobre las actividades que desarrollará y los objetivos de dichas actividades, marcando algunas diferencias entre la práctica profesional y la extensión universitaria. 
contenía una propuesta de malla curricular, una estructura central de la carrera, sobre la base de la cual se trabajaría en el período 2014-2015 para dar forma acabada al nuevo plan de estudios.

Finalmente, la Resolución n 313/15 del HCD sanciona el nuevo plan de estudios, el cual entraría en vigencia en el año 2017. Tanto la propuesta de reforma como el nuevo plan de estudios serán analizados en el presente artículo, con la pretensión de establecer algunas diferencias entre la estructura inicialmente propuesta y los contenidos del plan definitivo.

\section{Algunas aproximaciones teóricas: conceptos y clasificaciones} Caracterizar y definir a la extensión universitaria es una tarea de enormes magnitudes. El término "extensión universitaria" fue concebido tradicionalmente para denominar un conjunto de actividades a través de las cuales la universidad comunicaba a la sociedad los adelantos científicos y contribuía de esa manera a elevar culturalmente al pueblo trabajador. De allí en adelante, la expresión fue conceptualizada de diversas maneras, incluyendo una gran diversidad de objetivos y actividades. Para Giangiacomo y otros, la extensión ha comprendido y comprende una gran diversidad y número de actividades:

"Las áreas de extensión de las universidades comprenden todo tipo de actividades: culturales, sociales, de asistencia técnica, de desarrollo tecnológico, de difusión, de capacitación, de formación profesional; actividades destinadas a poblaciones específicas tales como adultos mayores, jóvenes y niños en situaciones de vulnerabilidad, discapacitados; cooperación con organismos gubernamentales y no gubernamentales y actividades abiertas a la comunidad en general tanto educativas como culturales; acciones y programas destinados a la propia comunidad universitaria, como deportes, becas, orientación vocacional y ocupacional y asistencia a los estudiantes en aquellas cuestiones de orden social, no estrictamente académicos (...) albergues, pasantías y bolsas de trabajo. (...) La definición de extensión pareciera estar más vinculada a lo que no es, a lo que no tiene un definido espacio institucional". (2011:2)
Coincidentemente, Vaccarezza considera que el concepto de extensión no se encuentra estabilizado ni dentro de una misma organización o discurso institucional, "situación alimentada, por demás, por la falta de regulación de sus actividades, fines, objetivos y modalidades" (2015:5). La expresión "extensión universitaria" ha sido cuestionada y sustituida —o al menos eso se intentó-; sin embargo, por diversas razones ha mantenido su predominio. La diversidad de significados que tiene y de actividades que comprende marca como una característica central la polisemia del término. Con esta polisemia como punto de partida, Vaccarezza (2015) ha elaborado un esquema que clasifica los significados de las funciones de la extensión universitaria según estén centradas en la universidad o en el mundo externo a ella. Entre las centradas en el mundo externo enumera: 1) contribuir a la elevación social y cultural de la población que no tiene acceso al conocimiento; 2) cumplir con el compromiso moral frente a la injusticia de una sociedad desigual; 3) brindar servicios para la satisfacción de necesidades o la solución de problemas sociales; 4) orientar y apoyar a la población en la presión al Estado para el cumplimiento de funciones de política social; 5) mostrar un modelo alternativo de acción profesional; 6) la extensión como efecto de demostración a la política del Estado; y 7) para insertar a la universidad como proveedora de conocimientosmercancía y en procesos de innovación productiva. Entre aquellas funciones que se centran en la universidad enumera: 1) devolver a la sociedad parte del esfuerzo que ésta hace para sostener la universidad; 2) acercar la universidad a la realidad social para abrir el conocimiento de los egresados y estimular su sensibilidad social; 3) permitir un ámbito de práctica social del alumnado; 4) identificar temas relevantes a estudiar/investigar/reflexionar por parte de la universidad; 5) demostrar un modelo alternativo de acción profesional: 6) la extensión como efecto de demostración de la política del Estado; 7) el ejercicio de extensión como banco de prueba y/o fuente de información en el proceso de investigación; y 8) extensión de beneficios a los estudiantes universitarios.

La clasificación realizada por Vacarezza nos permitirá analizar qué tipos de funciones contempla la Facultad como fundamentos para incluir la extensión universitaria en el nuevo plan de estudios. ${ }^{5}$
5) En el año 2012, en el marco del XIII Congreso Nacional y III Latinoamericano de Sociología Jurídica desarrollado en la Universidad Nacional de Río

Negro (UNRN), elaboramos un trabajo denominado "La extensión universitaria en los planes de estudio de las carreras de abogacía". Allí reflexionamos sobre la inclusión de la extensión en los planes de estudio de las carreras de Abogacía de universidades públicas nacionales. En ese entonces, solo la
UNRN y la Universidad Nacional del Centro de la Provincia de Buenos Aires (UniCen) incluía la extensión en el plan de estudios de la carrera de Abogacía, en ambos casos como una asignatura diferente a la práctica profesional. Cuando elaboramos la ponencia, la FCJyS había iniciado el proceso de análisis, debate y formulación de propuestas para la reforma del plan.

En razón de ello, solo pudimos esbozar una breve opinión sobre las propuestas que habíamos leído. En la mayoría de los casos se pensaba la inclusión de la extensión como un espacio para la práctica profesional, focalizado en la formación del estudiante. En este trabajo y otros (2010, 2012 y 2013) construimos una serie de preguntas como guía para analizar las modalidades a través de las cuales las facultades habían incluido la extensión en sus planes de estudio. Esas preguntas fueron el germen para la construcción de las categorías teórico-metodológicas desarrolladas posteriormente. Sin embargo, esas preguntas-categorías fueron pensadas para analizar documentos que establecen detalles sobre el funcionamiento de los espacios. En razón de ello, en el presente trabajo optamos por utilizar el esquema presentado por Vaccarezza, en tanto nos permite elaborar consideraciones más generales. 


\title{
64
}

\author{
la Responsabilidad Social Universitaria \\ (RSU) animará la implementación \\ del espacio de Enseñanza Práctica \\ por su "capacidad de poner en \\ práctica principios y valores por \\ medio de cuatro procesos clave de \\ toda universidad: gestión, docencia, \\ investigación y extensión"
}

\section{La propuesta de reforma del plan de estudios}

La Resolución $n^{\circ}$ 336/13 establece en su Anexo I una malla curricular compuesta por tres grandes bloques: introductorio, disciplinar y de orientación profesional. A estos bloques centrales, se les suma el llamado "Complemento de la formación" que incluye taller de lectocomprensión en idiomas extranjeros, el seminario de la carrera y un espacio de enseñanza práctica. En este espacio se propone:

"integrar a la malla curricular la experiencia educativa de los Consultorios Jurídicos Gratuitos, las Clínicas Jurídicas y otros Programas de la Secretaría de Extensión, más la posibilidad de hacer prácticas en organismos públicos y privados y en relación a los MARC - Medios Alternativos de Resolución de Conflictosen el Centro de Mediación de la FCJyS. En definitiva: curricularizar la extensión". (FCJyS, 2013:23)

De lo manifestado se desprenden dos aspectos interesantes. En principio, es importante la idea de "curricularizar la extensión", lo cual parece expresado casi como un objetivo central del espacio de Enseñanza Práctica. Asimismo, la idea de pensar en la "experiencia educativa" de los programas de extensión, como diferente de la posibilidad de hacer prácticas en organismos, parece establecer una mínima diferencia entre la experiencia educativa de la extensión universitaria y la realización de prácticas profesionales. A partir de allí, sin embargo, las referencias comienzan a centrarse exclusivamente en la idea de práctica, diluyéndose las referencias a la extensión universitaria. La Facultad prevé ofrecer al estudiante un espacio de práctica en escenario y tiempos reales. Para ello, se propone asignarlos en forma rotativa y alternativa a los consultorios, clínicas, proyectos de extensión y organismos con los que se tiene convenio, con la idea de que los grupos "no turben las tareas de los lugares en donde practicaran" (FCJyS, 2013:25). Los proyectos de extensión se comienzan a configurar como espacios que permiten "un ámbito de práctica social del alumnado", función centrada en la propia universidad (Vaccarezza, 2015:6). El documento establece que la configuración definitiva de la práctica dependerá del intercambio entre los docentes de las actuales prácticas y los "especialistas en extensión". Sería interesante saber qué canales se establecerán para viabilizar este diálogo y qué aspectos se tendrán en cuenta para tomar las decisiones correspondientes, considerando que este espacio de intercambio tan ameno suele transformarse en un campo de batalla disciplinar.

Retomando la idea de extensión universitaria, el documento propone trabajar con la metodología del "aprendizaje servicio" en tanto esa metodología "integra las dos dimensiones: el vínculo con la sociedad a través del servicio comunitario; y la formación integral de los miembros del Programa, resaltando los valores implícitos en el servicio" (FCJyS, 2013:25).

Es recién aquí que la curricularización planteada se liga a una función centrada en el mundo externo a la universidad: "brindar servicios para la satisfacción de necesidades o la solución de problemas sociales". Al mismo tiempo, y considerando la idea de las dos dimensiones, se destaca la formación integral de los miembros del programa, en los términos de Vacarezza hablamos de una función centrada en la universidad: "acercar la universidad a la realidad social para abrir el conocimiento de los egresados y estimular su sensibilidad social" (2015:6).

Hacia el final del documento se establece que la noción de Responsabilidad Social Universitaria (RSU) animará la implementación del espacio de Enseñanza Práctica por su "capacidad de poner en práctica principios y valores por medio de cuatro procesos clave de toda universidad: gestión, docencia, investigación y extensión" (FCJyS, 2013:26).

Más adelante, intentaremos observar si la Facultad integra algunos de estos conceptos —como la RSU o el aprendizaje servicio—en el nuevo esquema de la carrera. 
La propuesta de reforma del plan de estudios comenzaba con una idea muy fuerte: curricularizar la extensión. Esa curricularización parecía estar orientada por fundamentos ligados a funciones de la extensión que se centran en la propia universidad. Casi todas las referencias del documento con las excepciones marcadas suelen referirse a la idea de práctica y a los programas de extensión como centros de práctica.

A continuación, analizaremos la forma definitiva que adquirió el nuevo Plan de Estudios y el lugar que se reservó a la extensión universitaria.

\section{El nuevo Plan de Estudios}

La Resolución n³13/15 organiza el nuevo Plan de Estudios de la carrera en cuatro bloques: a) Formación General e Introductoria,

b) Formación Disciplinar, c) Orientación Profesional, y d) Formación para la Práctica Profesional. La formación práctica, que en la propuesta de reforma era una suerte de complemento, en el plan definitivo constituye uno de los cuatro bloques que conforman el Plan de Estudios. El bloque de la formación para la práctica prevé 412 horas de prácticas en escenarios y tiempos reales y está conformado por cuatro espacios diferentes: 1) Formación Práctica al interior de las asignaturas, 2) Adaptaciones Procesales Penales y Civiles, 3) Formación Práctica en temáticas específicas, y 4) Formación Práctica en situaciones reales (FPSR) / Prácticas Pre-Profesionales Supervisadas (PPS), fijándose para estas últimas una carga de 172 horas. Se establece una enumeración no taxativa de espacios donde podrán desarrollarse las FPSR/PPS, en tanto se encuentra aquí la primera mención concreta sobre actividades vinculadas a la extensión universitaria. Los ámbitos donde podrán desarrollarse las PPS son:

“1) Proyectos de Extensión, conforme existen actualmente y todos aquellos que en el futuro puedan desarrollarse. ${ }^{6}$

2) Prácticas Formativas Supervisadas en organismos públicos conveniados por la Facultad.

3) Prácticas Formativas Supervisadas en organizaciones privadas convenidas por la Facultad.
4) Prácticas Formativas en Estudios Jurídicos que se ajusten a la normativa que el $\mathrm{HCD}^{7}$ oportunamente establecerá para la firma de convenios con la Facultad.

5) Prácticas Formativas en el Centro de Mediación de la Facultad.

6) Prácticas en Centros de Mediación privados o públicos que hayan firmado convenios con la Facultad.

7) Proyectos de Investigación acreditados de nuestra Facultad de Ciencias Jurídicas y Sociales y otros en los que nuestros docentes investigadores sean parte y se desarrollen en agencias reconocidas de investigación.

8) Institutos, Centros de Investigación y Observatorios de la Facultad de Ciencias Jurídicas y Sociales que, a partir de sus funciones específicas, tengan como objetivos la investigación y producción de contenidos académicos.

9) Competencias estudiantiles de litigios simulados en diferentes áreas temáticas.

10) Talleres de práctica profesional

11) Programa de Educación en Contextos de Encierro.

12) Prácticas Formativas en Escribanías que se ajusten a la normativa que el HCD oportunamente establecerá para la firma de convenios con la Facultad.

13) Cualquier organismo que pudiera ofrecer un espacio propicio para la práctica conforme los requisitos definidos por el HCD.

14) Cualquier otra modalidad que se proponga y cuente con la aprobación del HCD”. (FCJyS, 2015:42)

La idea de la curricularización de la extensión, la consideración de los proyectos de extensión como experiencias educativas valiosas, la utilización de la metodología de aprendizaje-servicio y la noción de RSU han desaparecido en el nuevo documento. En este Plan de Estudios los proyectos de extensión de la Facultad constituyen uno de los tantos ámbitos entre los cuales el estudiante podrá optar como espacio para desarrollar la Formación Práctica en Situaciones Reales.

Las menciones a la extensión universitaria en el resto del documento son menores, y generalmente como expresiones
6) La Facultad cuenta actualmente con 16 programas dependientes de la Secretaría de Extensión Universitaria en los cuales los estudiantes podrían realizar sus FPSR/PPS. Ellos son: 1)

Consultorios Jurídicos Gratuitos, 2) Clínicas Jurídicas, 3) Unidad de Atención en Conflictos Juveniles, 4) Derecho a la salud: HIV y padecimientos mentales, 5) Derechos de las personas con padecimientos mentales, 6) Centro de Atención a Víctimas de Violencia de Género, 7) Espacio Migrante, 8) Centro de Atención Jurídica Gratuita para Productores Agropecuarios Familiares, 9) Facilitadores Jurídicos de Inclusión, 10) Observatorio de Relaciones Económicas Internacionales, 11) Observatorio Fiscal, 12) Diversidad familiar y derecho de familias, 13) Niñez, Derechos Humanos y Políticas Públicas, 14) Observatorio de la Producción Hortícola de la Región del Gran La Plata, 15) Centro de Acción Urbano Ambiental,

16) Observatorio de litigación y juicio por jurado. En el caso de los Consulto- rios Jurídicos Gratuitos, el sitio web de la Facultad enumera 20 consultorios. Por su parte, el Programa de Clínicas Jurídicas cuenta con ocho áreas o comisiones temáticas que conforman grupos de trabajo distintos: Derecho del Consumidor, Derecho Ambiental, Derechos Humanos, Derecho de Acceso a la Información Pública, Derecho Social, Hábitat, Arroyo El Gato e Interés Público.
7) El Honorable Consejo Directivo es el órgano de gobierno de la Facultad. Cumple la función de dictar disposiciones de carácter general en materia didáctica, disciplinaria y administrativa. Elige al decano y al vicedecano, formula y modifica el plan de estudios de la carrera, designa a los profesores como también a los secretarios de la Facultad a propuesta del decano, interviene en grado de apelación por los recursos administrativos que se interpongan, entre otras funciones. 


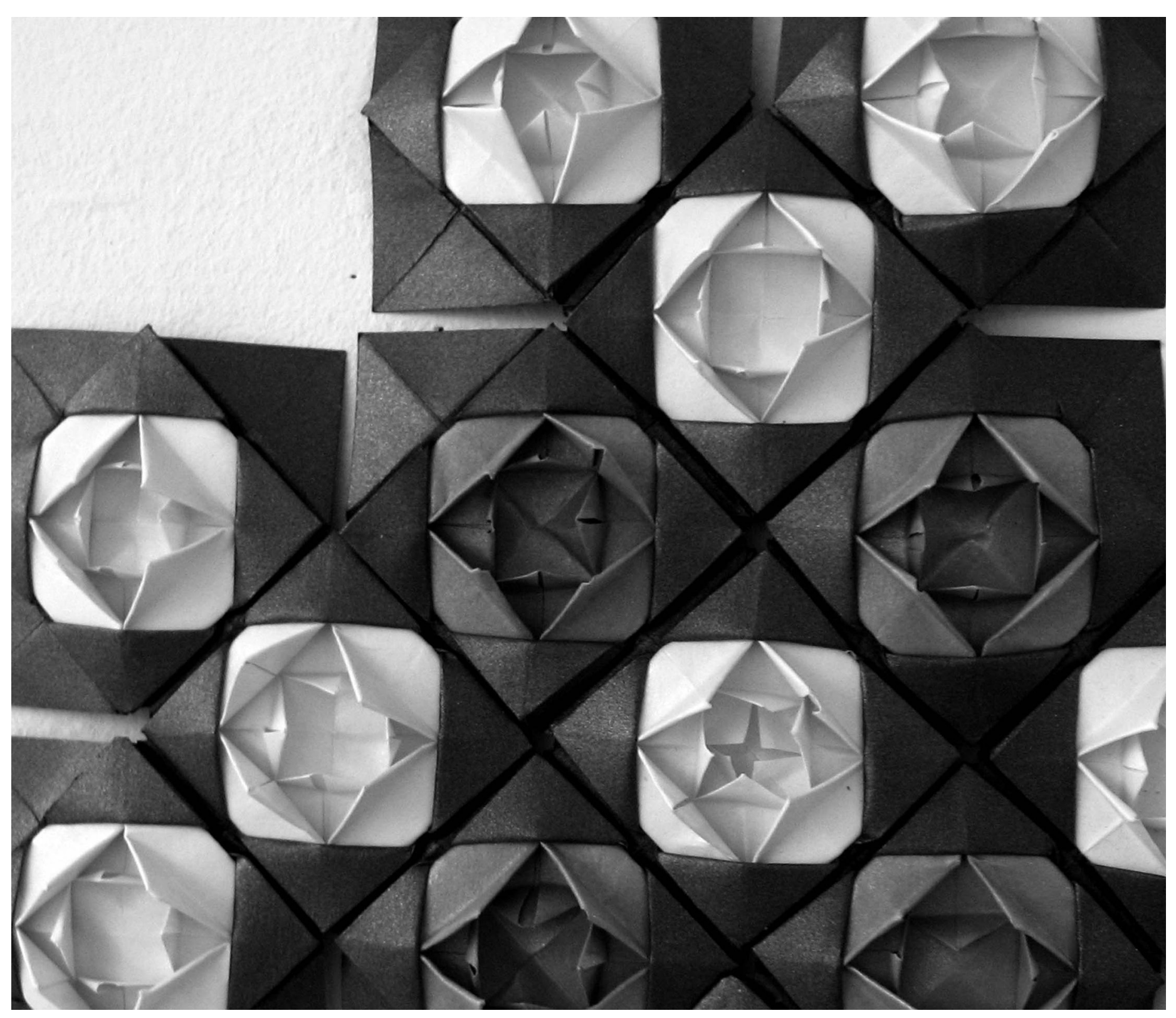

(c) Cecilia lucc 


\section{6}

la extensión universitaria debe ser

necesariamente crítica, no solo como

una evaluación del presente "sino

crítica en la medida en que trabaja en

la dirección de una nueva existencia" donde se destaca la necesidad de promover una integración de las funciones de la universidad que no tiene correlato con lo establecido en el nuevo Plan de Estudios, en el cual la extensión universitaria ha desaparecido. Otra mención aislada se incluye en la "Nueva normativa para la elaboración de programas de espacios curriculares en el marco de la reforma del Plan de Estudios" (FCJyS, 2015:23). Allí se consigna como requisito para la aprobación de los programas la integración de prácticas, actividades de investigación y de extensión. De alguna manera, pareciera que la Facultad —en el nuevo Plan de Estudios- le pasa la responsabilidad a los docentes, y éstos serían, al interior de cada asignatura, los encargados de integrar las actividades de extensión.

En el trayecto de la propuesta de reforma sancionada en el año 2013 hasta el nuevo plan del año 2015, la extensión pasó de ser una experiencia posible a una experiencia poco probable. De la idea de curricularizar la extensión y utilizar la metodología del aprendizajeservicio pasamos a ofrecer los proyectos de extensión como uno de los tantos ámbitos de práctica, borrando toda mención de la actividad extensionista. Pese a este retroceso, consideramos que la Facultad aún tiene la posibilidad de tomar ciertas decisiones que conduzcan hacia una curricularización fáctica de la extensión, ya sea mediante el establecimiento de algunas modificaciones reglamentarias o la utilización de su aparato publicitario para resaltar la multiplicidad y diversidad de experiencias que puede aportar al estudiante la oferta de proyectos de extensión en la FCJyS. Creemos que la reforma del Plan de Estudios era la oportunidad propicia para integrar las prácticas de extensión a la currícula y construir una asignatura que combinara la formación teórica sobre contenidos básicos de la extensión universitaria y las actividades en el terreno, es decir, una asignatura que promoviera una verdadera praxis extensionista. Como hemos visto, algunas ideas interesantes se manifestaban tempranamente en la propuesta de reforma del año 2013, pero las modificaciones que sufrió y que derivaron en el nuevo plan de estudios no brindan un panorama muy alentador al respecto.

\section{De recuerdos y reflexiones}

En mayo del año 2011, en el marco de una estadía de investigación, lleve a cabo una entrevista a los coordinadores de la Unidad de Proyectos del Servicio Central de Extensión y Actividades en el Medio (SCEAM) de la Universidad de La Republica (UdeLaR, Uruguay). El objetivo de la entrevista era conocer los lineamientos básicos de la extensión universitaria en la UdeLaR, tarea de investigación que realizaba en el marco del proyecto UNLP J101 "Las nuevas configuraciones del campo de la formación del abogado en Argentina: instituciones, planes de estudios y prácticas 
profesionales". La entrevista mencionada fue muy rica, pero hubo particularmente un detalle, una frase que me llamo la atención. Hablando sobre la inclusión de la extensión en los planes de estudio, Alicia Migliario, una de las coordinadoras, dijo "el problema con la Facultad de Derecho es que no sabe distinguir la práctica formativa de la actividad de extensión, para ellos hacer práctica es hacer extensión".

Claramente esta frase es polémica. Utilizarla en este artículo puede sonar hasta contradictorio, si consideramos que en el esquema de Vacarezza una de las funciones de la extensión que se centra en la universidad es la de "permitir un ámbito de práctica social del alumnado" (2015:6). Siguiendo este razonamiento, deberíamos decirle a Alicia que está equivocada, que en realidad la Facultad de Derecho cuando hace práctica está haciendo extensión. Sin embargo, no estoy seguro que le podamos decir eso, es más, no estoy de acuerdo con decirle eso, creo que lo correcto sería decirle: "Tenés razón Alicia, la Facultad de Derecho no sabe distinguir". Pero el problema no radica en decidir si tiene razón o no, el problema radica en explicitar cuales son para nosotros las preguntas que debe hacerse la extensión universitaria y cuáles son las respuestas que la diferencian de la práctica profesional. ¿Qué sujetos participan del espacio de extensión? ¿Cuáles son los perfiles y características que poseen? ¿Cómo participan? ¿Cómo emergió el espacio? ¿Cuáles son sus objetivos? ¿Qué concepciones atraviesan su planificación y desarrollo? ¿Cuáles son los contenidos y las actividades desarrolladas? ¿Se sistematizan las experiencias? ¿Quién y dónde utiliza ese conocimiento? ¿Cómo se utiliza? Creemos que partiendo de estas preguntas se podría plantear un debate acerca de cómo se desarrolla efectivamente la extensión universitaria, cuáles son sus objetivos reales y qué realidades transforma. Y quizás, a partir de esos diagnósticos, se podría dar mayor complejidad a las problemáticas abordadas por los espacios de extensión e incluir más sujetos para multiplicar las voces que habitan los programas de extensión universitaria.

La extensión universitaria debe ser necesariamente crítica, no solo como una evaluación del presente "sino crítica en la medida en que trabaja en la dirección de una nueva existencia" (Quinney en Wolkmer, 2000:5). En el mundo del Derecho, la extensión universitaria tiene la obligación de cuestionar las formas jurídicas y sociales dominantes, impulsando y construyendo colectivamente prácticas e ideas emancipadoras dentro y fuera del campo jurídico que representen alternativas viables al estado de cosas existente (García Villegas y Rodríguez, 2003).

Con este potencial en el horizonte, la extensión universitaria debe integrarse a la currícula del Derecho para sacudir sus telarañas, para dar al estudiante las armas que le permitan derrocar al individualismo reinante y así, al creer en el otro, lo inviten a sumergirse en la aventura de lo colectivo.

\section{Referencias bibliográficas}

Facultad de Ciencias Jurídicas y Sociales (FCJyS) (2011). Autoevaluación Académico-Institucional de la Facultad de Ciencias Jurídicas y Sociales de la Universidad Nacional de La Plata. UNLP.

Facultad de Ciencias Jurídicas y Sociales (FCJyS) (2013). Propuesta de reforma del plan de estudios de la carrera de abogacía. FCJyS. UNLP.

Facultad de Ciencias Jurídicas y Sociales (FCJyS) (2015). Nuevo Plan de Estudios de la carrera de Abogacía. FCJyS. UNLP.

Furfaro, C. (2010). La alfabetización jurídica entre líneas: un estado del arte sobre la enseñanza del derecho en sectores no universitarios. En Actas del XI Congreso Nacional y I Latinoamericano de Sociología Jurídica. Buenos Aires: Facultad de Derecho. UBA.

Furfaro, C. (2012). La extensión en los planes de estudio de las carreras de abogacía. En XIII Congreso Nacional y III Latinoamericano de Sociología Jurídica. Viedma: UNRN.

Furfaro, C. (2013). Sobre la construcción de herramientas teórico-metodológicas para analizar y discutir la extensión universitaria. En Crítica Jurídica. Revista Latinoamericana de Política, Filosofía y Derecho, 30 (36), 125-149. México: UNAM. García Villegas, M. y Rodríguez, C. (2003). Derecho y Sociedad en América Latina: Un debate sobre los estudios jurídicos críticos. Bogotá: ILSA.

Giangiacomo, G. y otros (2011). La Actividad de Extensión ¿función subalterna de la universidad? Un análisis desde las universidades del conurbano bonaerense. En Arias, A.; Beovide, A. y García Godoy, B. Políticas Públicas y Trabajo Social. Aportes para la reconstrucción de lo público. Buenos Aires: UBA Sociales. Vacarezza, L. (2015). Apropiación social e hibridación de conocimientos en los procesos de extensión universitaria. En Cuestiones de Sociología, (12). La Plata: UNLP. Wolkmer, A. (2000). Ideología, estado e direito. Revista dos Tribunais ( $3^{\circ}$ ed.). San Pablo. 